\title{
Double Probe Diagnosis of Surface-Wave Sustained Plasma Column
}

\author{
Jian $\mathrm{LI}^{1,2, a}$, Kun WANG ${ }^{1,2, b}$, Shi-qing WANG ${ }^{1,2, c}$ \\ ${ }^{1}$ Southwestern Institute of Physics, Chengdu, 610041, China \\ ${ }^{2}$ Engineering and Technical College of Chengdu University of Technology, Leshan, 614007, China \\ a lijwk@126.com, ${ }^{\mathrm{b}}$ wangkun6236@sina.com, ${ }^{\mathrm{c}}$ wsq6222@126.com
}

Keywords: Surface-Wave sustained plasma, ion density, electron temperature, plasma antennas.

\begin{abstract}
A technique has been developed to diagnose the plasma column generated with Surface-Wave sustained plasma (SWP) source under different conditions. Principal physical properties, ion density and electron temperature were studied. Influence of several factors, such as the microwave power, the gas pressure on the ion density, the electron temperature was studied. The axial and radial distribution of electron temperature and ion density was also researched.

The results show the Surface-Wave sustained plasma column is able to generate plasma of about 3 ev electron temperature and $1011 \mathrm{~cm}-3$ ion density. It is found in the experiment that at $2 \mathrm{~Pa}$ in gas pressure and $60 \mathrm{~W}$ in input power, the ion density and electron temperature are up to $6.8 \times 1017 \mathrm{~m}-3$ and $2.8 \mathrm{ev}$ at $200 \mathrm{~mm}, 1.5 \times 1017 \mathrm{~m}-3$ and $2.2 \mathrm{ev}$ at $1000 \mathrm{~mm}$ from the base. It is also found that the physical properties can be changed by adjusting the microwave power and the pressure. So, the Surface-Wave sustained plasma column is suit for the application of plasma antennas and other relative applications.
\end{abstract}

\section{Introduction}

Microwave and RF plasmas are finding increasing use in many fields. [1] A plasma column is constructed from glass tube filled with low pressure gases with the SWP source. This discharge style has many merits in plasma antennas [2]. The principal properties of the plasma, ion density and electron temperature have much influence on the application of plasma antennas [3, 4].

The diagnosis and the diagnosis method of the surface wave sustained plasma are not new [5-7]. However the diagnosis of the plasma column sustained by electromagnetic surface-wave, which is suit for the plasma antennas and lamps, is not done yet. So, it is necessary to study the properties under different conditions to guide the research in plasma antennas and plasma lamps.

This paper mainly researches in the diagnosis of SWP plasma column. The Langmuir Probe double probe is used as the diagnosis technique in the whole paper. The influence of microwave power, the gas pressure on the ion density, the electron temperature was studied. The axial and radial distribution of electron temperature and ion density was also researched.

\section{Experimental Setup}

Figure 1 shows the schematic of the surface-wave sustained plasma experimental apparatus. It contains microwave generator, the glass vacuum room and the vacuum systems. LC-box [1] is regarded as the pump method. A copper cylindrical sleeve wrapped around the base of an electrodeless tube containing a low-pressure noble gas (typically, argon at $\sim 10$ mTorr). A matching network was employed to match the coupler to the generator. The wave generator produce $100 \mathrm{MHz}$ microwave, passing through the glass window, and getting into the vacuum room to excite the plasma. The plasma tube, with $24 \mathrm{~mm}$ in diameter and $1200 \mathrm{~mm}$ in length, is made of glass. The apparatus can produce high density plasma without magnetic field. By adjusting the button of the wave generator, the reflected power can be changed. The base vacuum of the setup is $4 \times 10^{-3} \mathrm{~Pa}$. High purity gases act as the working gases. The gases were controlled with MFC. The input power of the wave generator is 
tested by the power meter. The power is $20 \sim 80 \mathrm{~W}$. The Langmuir double probe which locates at every $200 \mathrm{~mm}$ from the base is made of tungsten and glass. The size of the tungsten lines in the double probe is $0.5 \mathrm{~mm}$ in diameter and $6 \mathrm{~mm}$ in length. The depth into the plasma can be changed by moving the probe tubes. The data can be attained from the V-I Figureures in different conditions.

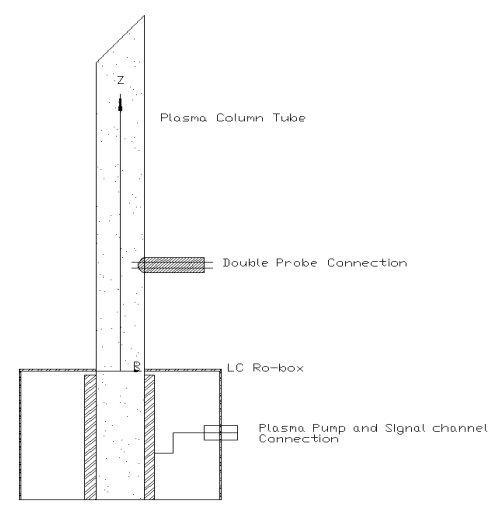

Figure 1. Experimental arrangement

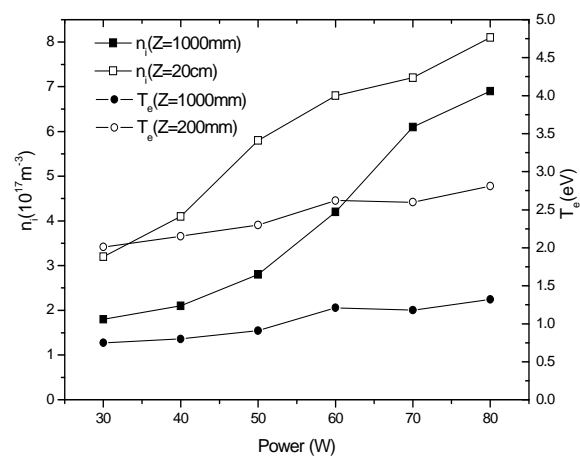

Figure 2. The measured ion density and electron temperature as a function of the power at $Z=200 \mathrm{~mm}$ and $\mathrm{Z}=1000 \mathrm{~mm}$

\section{Results and discussion}

All the diagnosis in this paper is tested by the Langmuir double probe. The method is one of the most important and frequently used plasma diagnostic techniques. It involves the measurement of electron and ion currents to a pair of small metal electrodes as different voltages are applied to the metal lines of the double probe. This yields a curve called the double probe characteristic of the plasma.

The electron temperature and ion density in the plasma can be calculated from the double probe characteristic [7-9]

$$
\mathrm{T}_{\mathrm{e}}=\frac{e}{4 k} \frac{I_{i 01}+I_{i 02}}{\frac{d I_{D}}{d V_{D}}-0.62 S} \quad \quad(1) ; \quad \mathrm{N}_{\mathrm{i}}=\frac{I_{i 0}}{\alpha e Z \sqrt{\frac{2 k T_{e}}{m_{i}}} A_{s}}
$$

Where Ii0, I01 and Ii02 are the saturated currents of the double probe, $\left.\frac{\mathrm{dI}_{\mathrm{D}}}{\mathrm{dV}_{\mathrm{D}}}\right|_{I_{D}=0}$ and $\mathrm{S}$ are the slope of the V-I characteristic when ID $=0$ and the current is saturated, . is the coefficient which ranges from 0.4 to $1, \mathrm{mi}$ is the relative mass of the gas and As is the surface area of the tungsten lines in the double probe.

Microwave Power and the ion density and electron temperature.Keeping the air pressure of the glass tube unchanged, the double was put at the fixed position $(Z=200 \mathrm{~mm}, R=12 \mathrm{~mm})$. The ion density and electron temperature were tested while the input microwave power changed. The reflected power was adjusted to the minimum each time. Figure 2 shows the data for a $1200 \mathrm{~mm}$ plasma tube with $24 \mathrm{~mm}$ in diameter driven at $100 \mathrm{MHz}$. The wave is capacitive couple by a sleeve of $50 \mathrm{~mm}$ in length as shown in Figure 1.

The electron temperature and ion density both increased with the power increasing. The increment of the microwave power leads to the increasing of the electric field at the edge of the vacuum room, which caused the increase of the hot electrons and more neutral gas molecules were ionized. From the data, it is obvious that the electron temperature and ion density can be changed by adjusting the input microwave power.

Electron temperature and Ion density vs. the gas pressure. Figure 3 shows that the temperature and ion density while the pressure change from $1 \mathrm{~Pa}$ to $60 \mathrm{~Pa}$. The input power is $60 \mathrm{~W}$ and the double probe was put at $200 \mathrm{~mm}$ from the base of the column. With the pressure increasing, the electron temperature and ion density decreased. 
The frequency of elastic and inelastic pumping increased with the pressure increasing. So the electrons can't acquire enough time to accelerate to a higher speed. The electrons with lower energy are not able to have more neutral atoms ionized [10-14], which caused the electron temperature and the ion density decreased. The pressure has much effect on the plasma resistance, too. When the pressure changed, the reflected power changed.

From the data, it is obvious that at the lower pressure, which is lower than $5 \mathrm{~Pa}$, the ionization efficiency is higher than that at higher pressure for our apparatus. This shows that the ionization efficiency at the pressure of lower than $5 \mathrm{~Pa}$, is higher than that at higher pressure.

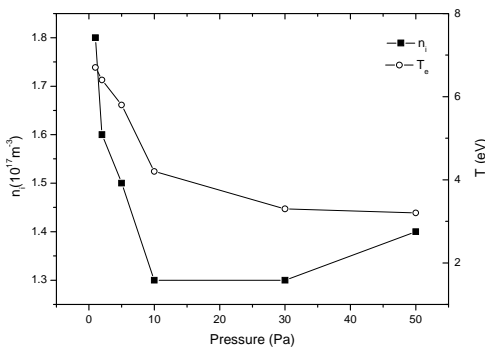

(a)

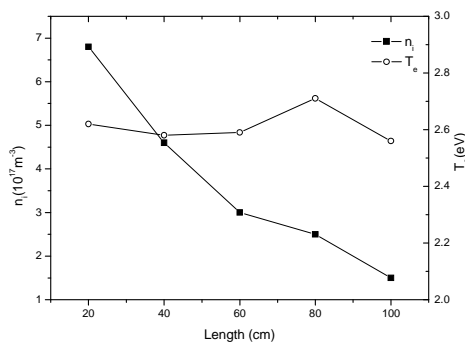

(b)

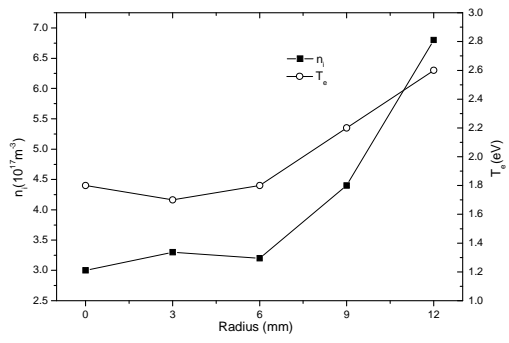

(c)

Figure 3. (a)The measured ion density and electron temperature as a function of Pressure (b) The measured ion density and electron temperature as a function of the length (c) The measured average ion density and electron temperature as a function of Radius

The axial distribution of electron temperature and ion density.Fig. 3. (b)shows the average ion density and electron temperature measured along a plasma column driven by the microwave at 100 $\mathrm{MHz}$ in frequency and $80 \mathrm{~W}$ in power. The plasma extends along the full length of the tube. All the data here is tested at $Z=200 \mathrm{~mm}$. From the base $Z=0$ to the top $Z=1$, the ion density drop with $Z$ increase, while the electron temperature doesn't change.

The initial breakdown takes place in the discharge tube close to the launcher gap. The plasma column is launched at $Z=0$, and ends at $Z=1$, where the wave power drops below the level necessary to sustain the plasma [1]. The surface wave propagates until it is reflected back at the axial position. The microwave power is absorbed when it propagates. So from the power balance theory [2], the density is the function of the function of the input power which decreased along the plasma column. Therefore, the plasma density will drop along the plasma column. When there is no plasma, the surface wave cannot propagate and the plasma column ends.

The electron temperature Te doesn't change with $\mathrm{Z}$ increased. Te can be taken as the function of radius $\mathrm{R}$ and pressure $\mathrm{P}$ [2]. Therefore, the electron temperature doesn't change with the axial size change. This is in agreement to the results in [2] and [15].

The radial distribution of electron temperature and ion density. The radial distribution of the cross section average ion density is sketched in Fig. 3(c). The average ion density and electron temperature increase with the radius increases.

From above, $\mathrm{Te}$ is the function of pressure $\mathrm{P}$ and radius $\mathrm{R}$. When the pressure and the input power are fixed, Te will increase with the radius $\mathrm{R}$ increasing.

As the frequency of the driven source is much lower than that of the plasma, the plasma is like a metal, and the microwave can only propagate along the interface of the plasma. Therefore, with the radius increases, the higher microwave power density caused higher ion density and higher electron temperature of the plasma. This is why this kind of plasma is called surface wave sustained plasma.

\section{Conclusions}

A usable method of plasma diagnosis is introduced to diagnose the plasma column generated with Surface-Wave sustained plasma (SWP) source under different conditions. The principal physical properties, ion density and temperature were studied. The influence of the power, the pressure and the radial and axial distribution on the plasma physical properties is introduced and discussed. 
The results show the SWP column is able to generate plasma of about $3 \mathrm{ev}$ electron temperature and $1011 \mathrm{~cm}-3$ ion density. The ion density and electron temperature can be changed by adjusting the microwave power and the pressure. The corresponding cut-off frequency of the plasma column is up to $\mathrm{GHz}$, so it is fit for High-frequency communication. So it is suit for the application of plasma antennas and it is also suit for other applications, such plasma lamps.

\section{References}

[1] Moisan and Z Zakrzewski, Plasma Sources based on the propagation of electromagnetic surface waves, J. Phys. D 24, pp.1025-1034, 1991

[2] Jhon Phillip Rayner, Adrian Philip Whichllo, and Andrew Desmond Cheetham, Physical characteristics of plasma antennas, IEEE Transaction on Plasma vol. 32, no. 1, pp.269-281, 2004.

[3] Gerard G. Borg, Jeffrey H. Harris, Application of plasma columns to radiofrequency antennas, Applied Physics Letters vol. 74, no. 22, pp.3272-3274, 1999.

[4] Elsherbeni, A. Z. and A. A. Kishk, Modeling of cylindrical objects by circular dielectric and conducting cylinders, IEEE Trans. Antennas Propag., Vol. 40, pp.96-99, 1992.

[5] Liu, J., W.-Y. Yin, and S. He, A new defected ground structure and its application for miniaturized switchable antenna, Progress In Electromagnetics Research, Vol. 107, pp.115-128, 2010.

[6] Kamarudin, M. R. B., P. S. Hall, F. Colombel, and M. Himdi, Electronically switched beam disk-loaded monopole array antenna, Progress In Electromagnetics Research, Vol. 101, pp.339-347, 2010.

[7] Cheng, Z. F., Design and research on plasma microwave reflector, Center for Space Science and Applied Research, China, Beijing, 2010.

[8] Haleakala Research and Development, Inc., Commercial Smart Plasma Antenna Prototype, Brookfield, Massachusetts, USA, 2008.

[9] Mathew, J., R. A. Meger, R. F. Fernsler, D. P. Murphy, R. E. Pechacek, and W. M. Manheimer, "Electronically steerable plasma mirror based radar antenna," 10th International Conference on Antenna and Propagation, Vol. 436, 1469-1473, Apr. pp.14-17, 1997.

[10] F. F. Chen, Introduction to Plasma Physics, New York: Plenum Press, 1974.

[11] 1. Dwyer, T. J., J. R. Greig, D. P. Murphy, J. M. Perina, and R. E. Pechacek, "On the feasibility of using an atmospheric discharge plasma as an RF antenna," IEEE Trans. Antennas Propag., Vol. 32, pp.141-146, 1984.

[12] Brog, G. G., J. H. Harris, D. G. Miljak, and N. M. Martin, Application of plasma columns to radiofrequency antenn, Appl. Phys. Lett., Vol. 74, pp.3272-3274, 1999.

[13] Brog, G. G., et al., "Plasma as antenna: Theory, experiment and applications," Phys. Plasma., Vol. 7, pp.2198-2202, 2000.

[14] Rayner, J. P., A. P. Whichello, and A. D. Cheetham, "Physical characteristics of a plasma antenna," Proc. 11th Int. Conf. Plasma Physics, pp.392-395, Sydney, Australia, Jul. 2002.

[15] Fathy, A. E., A. Rosen, H. S. Owen, and F. Mc-Ginty, Silicon-based reconFigureurable antennas - Concepts, analysis, implementation and feasibility, IEEE Trans. Microwave Theory Tech., Vol. 51, pp. 1650-1661, 2003. 